\title{
The effect of Sacubitril/Valsartan on cardiac function and cardiac remodeling in patients with heart failure with reduced ejection fraction
}

\author{
Fangyuan Chen, Gang Tian, Xiaojun Bai, Juanli Li, Zuyi Yuan \\ Department of Cardiovascular Medicine, First Affiliated Hospital of Medical College, Xi'an Jiaotong University, Xi'an, China \\ Contributions: (I) Conception and design: F Chen; (II) Administrative support: Z Yuan; (III) Provision of study materials or patients: G Tian; (IV) \\ Collection and assembly of data: X Bai; (V) Data analysis and interpretation: J Li; (VI) Manuscript writing: All authors; (VII) Final approval of \\ manuscript: All authors. \\ Correspondence to: Fangyuan Chen. Department of Cardiovascular Medicine, First Affiliated Hospital of Medical College, Xi'an Jiaotong University, \\ 277 Yanta West Road, Xi’an, China. Email: chenfy-608@163.com.
}

\begin{abstract}
Background: The aim of this study was to observe the effect of Sacubitril/Valsartan on cardiac function and remodeling in patients with heart failure with reduced ejection fraction (HFrEF).

Methods: A total of 120 patients with HFrEF were selected and given standard heart failure treatment according to the 2017 ACC/AHA/HFSA guidelines. Regardless of whether patients had taken AngiotensinConverting Enzyme Inhibitors/Angiotensin Receptor Blockers (ACEI/ARB) medications previously, after admission they were treated with the minimum effective dose of Sacubitril/Valsartan according to their blood pressure reading. Baseline clinical data were recorded and patients were followed up at 1, 3, 6, 9, and 12 months post discharge, during which time the dose of Sacubitril/Valsartan was gradually increased to the maximum tolerated dose (dose range $25-200 \mathrm{mg}$ /twice daily). During follow-up, N-terminal pro-brain natriuretic peptide (NT-proBNP), left ventricular ejection fraction (LVEF), left ventricular end diastolic diameter (LVEDD), left ventricular end systolic diameter (LVESD), and left atrium diameter (LAD) were monitored; a 6-minute walking test and Kansas City Cardiomyopathy Questionnaire (KCCQ) scores were recorded; and adverse reactions were collected.
\end{abstract}

Results: Over the course of the 12-month follow-up, the plasma concentrations of NT-proBNP were significantly reduced compared with the baseline, and the longer the follow-up time, the lower the NTproBNP levels were $(\mathrm{P}<0.05)$. Similarly, LVEDD, LVESD and LAD were significantly smaller at 12 months than at baseline, and the longer the follow-up time, the smaller the internal diameter was $(\mathrm{P}<0.05)$. The LVEF, 6-minute walking distance and KCCQ scores increased significantly from baseline $(\mathrm{P}<0.05)$, whereas eGFR and serum potassium levels showed no significant change compared with the baseline.

Conclusions: Sacubitril/Valsartan demonstrated a remarkable ability to improve cardiac function and to control cardiac remodeling with a high degree of safety in patients with HFrEF.

Keywords: Sacubitril/Valsartan; heart failure with reduced ejection fraction (HFrEF); cardiac remodeling

Submitted Dec 14, 2020. Accepted for publication Aug 05, 2021.

doi: $10.21037 /$ apm-21-157

View this article at: https://dx.doi.org/10.21037/apm-21-157

\section{Introduction}

Heart failure (HF) is the end stage of various types of cardiovascular disease. The prevalence of HF continues to rise, and the rates of readmission and mortality also continue to increase significantly (1). Cardiac remodeling is the basic mechanism underlying the progression of $\mathrm{HF}$, accompanied by the activation of neuroendocrine and various cytokines $(2,3)$. With the development of HF, these 
mechanisms promote each other to form a vicious circle and induce the deterioration of heart function $(4,5)$. There are three types of $\mathrm{HF}$, heart failure with preserved ejection fraction (HFpEF), heart failure with mid-range ejection fraction (HFmrEF), and heart failure with reduced ejection fraction (HFrEF). Of these, HFrEF has a worse prognosis. HFrEF refers to patients with symptoms and/or signs of $\mathrm{HF}$ and left ventricular ejection fraction (LVEF) $<40 \%$ (6).

At present, the effect and definite mechanism of $\mathrm{HF}$ is still unclear. With the development of HF, the heart function gradually changes from compensation to decompensation, leading to obvious symptoms and signs of heart failure. NT-proBNP is a significant neuroendocrine hormone decomposed from pro-BNP, which is composed by the ventricle of myocardial. With the aggravation of cardiac failure, the level of NT-proBNP is increased rapidly. Moreover, NT-proBNP is an important marker of myocardial cell damage and cardiac function. Not only that, the concentration of NT-probNP is higher and the half-life is longer, more stable and better reflects the severity of heart failure. Thus, NT-proBNP has apparently important guide in the diagnosis, treatment and prognosis of heart failure.

Sacubitril/Valsartan is a dual blocker of enkephalinase and the -angiotensin-aldosterone system (RAAS) (7). The large-scale PARADIGM-HF trial found that Sacubitril/ Valsartan reduced the risk of the primary end point (cardiovascular death, HF, and re-hospitalization) by $20 \%$, the risk of the secondary end point (all-cause death) by $16 \%$, and increased the Kansas City Cardiomyopathy Questionnaire (KCCQ) scores for patients with grade IIIV cardiac function compared with classic drugs such as enalapril commonly used to treat HF $(8,9)$. What's more, Sacubitril/Valsartan can significantly reduce the size of heart and cardiac remodeling was inhibited. The 2017 ACC/ AHA/HFSA guidelines for the diagnosis and treatment of HF outline the following indications for Sacubitril/ Valsartan treatment: For patients with NYHA grade IIIV and symptomatic HFrEF who can tolerate ACEI/ ARB treatment, it is recommended to replace ACEI/ARB with Sacubitril/Valsartan in order to further reduce the incidence rate and mortality of heart failure (10). However, there remains insufficient data and evidence in regard to outcomes for HFrEF patients treated with Sacubitril/ Valsartan regardless of whether they had received prior treatment with ACEI/ARBs. Some preliminary results from the PIONEER-HF and PROVE-HF studies have suggested that early administration of Sacubitril/Valsartan can benefit patients with heart failure regardless of a prior history of heart failure or previous ACEI/ARB treatment. This study aims to further investigate the effect of Sacubitril/ Valsartan on cardiac function and cardiac remodeling in HFrEF patients regardless of whether previous ACEI/ $A R B$ treatment was received or whether there was a previous history of HF. We present the following article in accordance with the TREND reporting checklist (available at https://dx.doi.org/10.21037/apm-21-157).

\section{Methods}

\section{Patients}

This was an open-label, nonrandomized, non-controlled, single center, dose-titration study of patients with HFrEF. According to the guidelines for HF to be identified. It has to satisfy the following basic conditions: (I) patients possess the symptoms and signs of HF; (II) left ventricular systolic function decreased with left ventricular social score reduced; (III) related cardiac structural lesions. This study conformed to the rules for the protection of human subjects and collected informed consent as reviewed and approved by an institutionally appointed committee. All participants gave written informed consent to participate in this study, and the study protocol conformed to the ethical guidelines of the Declaration of Helsinki (as revised in 2013). A total of 120 patients who were diagnosed with HFrEF and treated with Sacubitril/Valsartan were randomly selected from the Department of Cardiovascular Medicine, First Affiliated Hospital of Medical College, Xi'an Jiaotong University from May 2018 to November 2018. The study was approved by institutional ethics board of First Affiliated Hospital of Medical College, Xi'an Jiaotong University (No.:2019SF-114).

The baseline characteristics of the enrolled patients are shown in Table 1.

\section{Eligibility criteria}

Inclusion criteria were as follows: Male and female (nonchildbearing potential) patients (aged $>18$ years) with symptoms and/or signs of heart failure [defined as New York Heart Association (NYHA) functional grades II-IV], a documented LVEF of $\leq 40 \%$, and no contraindications to Sacubitril/Valsartan were screened for enrollment.

Patients were excluded if any of the following criteria were met: hemodynamic instability; use of a circulatory auxiliary circulation device; severe hepatic and renal 
Table 1 Baseline characteristics of included patients before treatment with Sacubitril/Valsartan

\begin{tabular}{|c|c|}
\hline Variable & $\begin{array}{c}\text { Mean } \pm \text { SD/number of } \\
\text { patients }(n=120)(\%)\end{array}$ \\
\hline Age (years) & $55.4 \pm 18.8$ \\
\hline Male & $82(68.3)$ \\
\hline Female & $38(31.7)$ \\
\hline NYHA II & $80(66.7)$ \\
\hline NYHA III & $35(29.2)$ \\
\hline NYHA IV & $5(4.1)$ \\
\hline Ischemic etiology & $74(61.6)$ \\
\hline Mean duration of heart failure (months) & 32 \\
\hline New-onset heart failure & $15(12.5)$ \\
\hline \multicolumn{2}{|l|}{ Guideline-recommended care } \\
\hline$\beta$-blocker & $65(54.2)$ \\
\hline RASI & $56(46.7)$ \\
\hline MRA & $78(65.0)$ \\
\hline CRT/CRTD & $3(2.5)$ \\
\hline \multicolumn{2}{|l|}{ ACEI/ARB administration } \\
\hline Never used & $29(24.2)$ \\
\hline Irregular use & $35(29.2)$ \\
\hline
\end{tabular}

NYHA, New York Heart Association; RASI, Renin Angiotensin System linhibitor; MRA, mineralocorticoid receptor antagonists; CRT, Cardiac Resynchronization Therapy; CRTD, Cardiac Resynchronization Therapy Defibrillator; ACEI, AngiotensinConverting Enzyme Inhibitors; ARB, angiotensin receptor blockers.

insufficiency (ALT $>120 \mathrm{U} / \mathrm{L}$ and eGFR $<30 \mathrm{~mL} / \mathrm{min}$ ); blood pressure $<100 / 60 \mathrm{mmHg}$; serum potassium $>5.3 \mathrm{mmol} / \mathrm{L}$; severe pulmonary hypertension; malignant arrhythmia; malignant tumor; a known history of hereditary or primary angioedema; serious adverse drug reactions or serious complications over the course of treatment.

\section{Study design}

An echocardiography was completed within 24 hours of admission. If LVEF was less than $40 \%$, the patients were treated with Sacubitril/Valsartan (Purchased). Dependent on the patient's blood pressure, Sacubitril/Valsartan was started at the minimum effective dose, and gradually adjusted to the maximum tolerated dose according to the patient's blood pressure at each follow-up. The dose range was $25-200 \mathrm{mg} /$ twice daily. Other therapeutic medications were administered to patients as needed in accordance with their general condition and the heart failure guidelines.

\section{Observation indices}

The biochemical index included renal function, electrolyte, and N-terminal pro-brain natriuretic peptide (NT-proBNP) levels, which were monitored on admission and during follow-up. Renal function and electrolytes were monitored by the Roche automatic biochemical immune assembly line. NT-proBNP was detected by fluorescence-enhanced immunochemiluminescence. All blood samples were sent to our central laboratory for testing.

Echocardiography was completed within 24 hours of admission, and jointly administered by two experienced staff from our department. The left ventricular end diastolic diameter (LVEDD), left ventricular end systolic diameter (LVESD), LVEF, and left atrial anterior posterior diameter (LAD) were measured by the modified Simpson method. The heart color Doppler ultrasound diagnostic system (Philips,) was used for all measurements.

The 6-minute walk test (11) is an exercise test of distance walked over a 6-minute time span, where walking distance $<150 \mathrm{~m}$ indicates severe cardiac insufficiency, $150-425 \mathrm{~m}$ indicates moderate insufficiency and 426-550 $\mathrm{m}$ indicates mild insufficiency.

The Kansas City Cardiomyopathy Questionnaire (KCCQ) (12) is a quality of life index consisting of 15 questions assessing the current activity tolerance and quality of life of patients with heart failure. The questionnaire can be completed in 4-6 minutes. Patients were instructed that there were no right or wrong answers and to complete the questionnaire truthfully according to their current situation.

\section{Follow-up}

The patients were followed up in the outpatient department at $1,3,6,9$, and 12 months post discharge, and the dosage of various treatment drugs for heart failure was gradually adjusted according to the patient's blood pressure, heart rate, general condition and adverse reactions.

\section{Statistical methods}

SPSS18.0 software was used for statistical analysis of data 
Table 2 Variations in the 6-minute walk test distance and KCCQ scores from baseline to 12 months in HFrEF treatment with Sacubitril/ Valsartan

\begin{tabular}{|c|c|c|c|c|c|}
\hline Time & Number of cases (n) & 6-minute walking distance $(\mathrm{m})$ & $\mathrm{P}$ & KCCQ score (score) & $\mathrm{P}$ \\
\hline 3 months & 105 & $350.9 \pm 65.7^{*}$ & $<0.05$ & $66.9 \pm 9.4^{*}$ & $<0.05$ \\
\hline 6 months & 102 & $430.8 \pm 45.6^{\#}$ & $<0.05$ & $70.6 \pm 11.6^{\#}$ & $<0.05$ \\
\hline 9 months & 100 & $500.5 \pm 60.6^{8}$ & $<0.05$ & $73.2 \pm 10.8^{8}$ & $<0.05$ \\
\hline
\end{tabular}

Note: compared with baseline, ${ }^{*}, \mathrm{P}<0.05$; compared with 3 months; ${ }^{*}, \mathrm{P}<0.05$; compared with 6 months; ${ }^{*}, \mathrm{P}<0.05$; compared with 9 months; ${ }^{\circledR}, \mathrm{P}<0.05$. KCCQ, Kansas City Cardiomyopathy Questionnaire; HFrEF, heart failure with reduced ejection fraction.

Table 3 Variation of NT-proBNP from baseline to the 12th month in EFrHF treatment with Sacubitril/Valsartan

\begin{tabular}{lccc}
\hline Time & Number of cases $(\mathrm{n})$ & NT-proBNP $(\mathrm{pg} / \mathrm{mL})$ & $\mathrm{P}$ \\
\hline Baseline & 120 & $4,200.5 \pm 2,056.8$ & - \\
1 month & 118 & $2,520.2 \pm 1,230.4^{*}$ & $<0.05$ \\
3 months & 105 & $1,485.7 \pm 566.7^{\#}$ & $<0.05$ \\
6 months & 102 & $856.2 \pm 335.6^{\&}$ & $<0.05$ \\
9 months & 100 & $488.6 \pm 225.3^{+}$ & $<0.05$ \\
12 months & 98 & $450.5 \pm 200.6^{\circledR}$ & $<0.05$ \\
\hline
\end{tabular}

Note: compared with baseline, ${ }^{*}, \mathrm{P}<0.05$; compared with 1st month, \#, $\mathrm{P}<0.05$; compared with 3rd month, \&, $\mathrm{P}<0.05$; compared with 6th month, ${ }^{+}, \mathrm{P}<0.05$; compared with 9th month, ${ }^{\circledR}, \mathrm{P}<0.05$. NT-proBNP, N-terminal pro-brain natriuretic peptide; $\mathrm{HFrEF}$, heart failure with reduced ejection fraction.

in each group. The independent-sample $t$ test was used for comparison between groups and Comparisons of data from multiple groups were conducted using the one-way analysis of variance (ANOVA).

\section{Results}

\section{The number of patients and baseline characteristics before treatment with Sacubitril/Valsartan}

A total of 120 patients with heart failure were enrolled according to the inclusion and exclusion criteria. The detailed patient characteristics are shown in Table 1.

\section{The occurrence of end point events and improvement of life quality after treatment with Sacubitril/Valsartan}

After treatment with Sacubitril/Valsartan, patients were followed up for 12 months. During the follow-up period, 5 patients died of cardiovascular events, 6 patients left the study, 7 patients were lost to follow-up, 2 patients stopped the drug due to hypotension, 2 patients stopped the drug due to angioedema, which left a total of 98 patients who completed the follow-up. Of these, there were 16 readmissions due to recurrent heart failure, 5 deaths due to cardiovascular events, and no deaths from other reasons. The patients' KCCQ baseline mean score of 63.7 increased to 73.2 , and the average walking distance in the 6-minute walking test increased from a baseline of 203.5 to $510.2 \mathrm{~m}$. Over the course of the 12-month follow-up, the KCCQ scores and walking distance increased gradually. There were significant differences among the 3, 6, 9, and 12 months follow-up periods (Table 2).

\section{Effect of Sacubitril/Valsartan on NT-proBNP concentrations}

The concentration of NT-proBNP decreased significantly from the initial treatment to the 1-month follow-up, which was the largest overall decline (an average value of 40\%). With the extension of the follow-up time, the level of NTproBNP decreased steadily over the 3, 6, 9, and 12 months time periods (Table 3).

\section{Changes of LVEF from baseline to 12 months following treatment with Sacubitril/Valsartan}

The mean value of LVEF had increased by $3.4 \%$ in 3 months, $8.7 \%$ in 6 months, $13.5 \%$ in 9 months, and $14.6 \%$ in 12 months. With the extension of the follow-up time, LVEF increased significantly, and the difference was statistically significant (Table 4). 
Table 4 Variation of LVEF from baseline to 12 months in HFrEF treatment with Sacubitril/Valsartan

\begin{tabular}{lccc}
\hline Time & Number of cases $(\mathrm{n})$ & EF $(\%)$ & $\mathrm{P}$ \\
\hline Baseline & 120 & $30.2 \pm 7.8$ & - \\
3 months & 105 & $33.6 \pm 5.6^{*}$ & $<0.05$ \\
6 months & 102 & $38.9 \pm 8.6^{\#}$ & $<0.05$ \\
9 months & 100 & $43.7 \pm 10.4^{\&}$ & $<0.05$ \\
12 months & 98 & $44.8 \pm 8.6^{\circledR}$ & $<0.05$ \\
\hline
\end{tabular}

Note: compared with baseline, ${ }^{*}, \mathrm{P}<0.05$; compared with 3 months, ", $\mathrm{P}<0.05$; compared with 6 months, ${ }^{\&}, \mathrm{P}<0.05$; compared with 9 months, ${ }^{\circledR}, \mathrm{P}<0.05$. LVEF, left ventricular ejection fraction; HFrEF, heart failure with reduced ejection fraction.

\section{The effect of Sacubitril/Valsartan treatment on cardiac remodeling from baseline to 12 months}

After 12 months' treatment with Sacubitril/Valsartan, the mean value of LVEDD was reduced from 78.6 to $57.6 \mathrm{~mm}$, LVESD was reduced from 60.5 to $42.3 \mathrm{~mm}$, and LAD was reduced from 41.3 to $31.2 \mathrm{~mm}$. Over the extended time of treatment, the size of the heart was significantly reduced, and cardiac remodeling was inhibited. The difference was statistically significant (Table 5).

\section{Adverse reactions to Sacubitril/Valsartan treatment from baseline to 12 months}

Of the adverse reactions recorded during the treatment time, hypotension and dizziness were recorded in 8 cases (6.7\%), but symptoms improved on reduction of the dosage. The incidence rates of renal function deterioration and hyperkalemia were $2.5 \%$ and $1.7 \%$ in 3 cases and 2 cases, respectively, but there was no significant change in the mean glomerular filtration rate (eGFR) and serum potassium levels before or after treatment (Table 6). The incidence of angioedema was $1.7 \%$ ( 2 cases), which normalized after discontinuation of treatment.

\section{Discussion}

HF is a complex, vicious circle process based on cardiac remodeling, which is activated and promoted by a variety of neuroendocrine humoral factors (13). As such, the mortality and readmission rates for HFrEF remain unacceptably high even when ACEI/ARBs, $\beta$-blockers, aldosterone receptor antagonists, diuretics, digoxin and other drugs are used as recommended by current guidelines $(14,15)$. Previous research has found that ameliorating cardiac remodeling can improve patients' quality of life and survival rate (16). It is suggested to pay attention to rest at ordinary times and have a balanced diet. What's more, patients need to do moderate exercise but avoid overexertion. Not only that, patients should keep enough sleep to avoid catching a cold and maintain their mood at ease. Last but not least, patients may insist on taking drugs and check regularly.

Sacubitril/Valsartan is more effective in improving cardiac function and exercise endurance in patients with heart failure. The most striking feature of heart failure is the activation of the renin-angiotensin-aldosterone system, which leads to cardiac myocyte abnormalities and myocardial dysfunction. Sacubitril/Valsartan is a sodium complex that acts as a dual inhibitor of enkephalin enzymes and angiotensin receptors, dilating blood vessels and promoting urinary sodium excretion, thereby lowering blood pressure and alleviating heart failure symptoms. The effect of valsartan on myocardial fibrosis and cardiac hypertrophy is more obvious, which can not only reverse cardiac remodeling, but also improve exercise endurance. Sacubitril/Valsartan can not only increase the level of natriuretic peptide, but also inhibit RAAS. More importantly, Sacubitril/Valsartan can significantly relieve myocardial injury and achieve the effect of treating heart failure. Sacubitril/Valsartan acts as an angiotensin receptor/enkephalin enzyme inhibitor. The large-scale PARADIGM-HF trial study found that, compared with enalapril, Sacubitril/Valsartan could significantly reduce cardiovascular mortality and incidence rate in HFrEF patients without significant safety concerns $(8,17)$. Therefore, Sacubitril/Valsartan is the most promising drug to emerge in the field of HF treatment in the past 20 years. The clinical benefits observed in the PARADIGM-HF study can be applied to a wide range of HF patients, even those who have previously received the best practice treatment $(18,19)$.

In terms of its pharmacological mechanism, Sacubitril/ Valsartan improves the prognosis of HF patients mainly through the activation of the natriuretic peptide system and the inhibition of the RAAS system (20). Thus, its functions include promotion of diuresis and sodium excretion, blood pressure reduction, vasodilation, aldosterone secretion increases, sympathetic system activity inhibition, and ultimately improvements in myocardial remodeling $(21,22)$. In our study, regardless of whether the patients used ACEI/ ARBs or not, they were treated with Sacubitril/Valsartan 
Table 5 Variation of LVEDD, LVESD and LAD from baseline to 12 months in HFrEF treatment with Sacubitril/Valsartan

\begin{tabular}{|c|c|c|c|c|c|c|c|}
\hline Time & Number of cases (n) & LVEDD (mm) & $\mathrm{P}$ & LVESD (mm) & $\mathrm{P}$ & LAD (mm) & $\mathrm{P}$ \\
\hline 3 months & 105 & $73.4 \pm 8.9^{*}$ & $<0.05$ & $54.3 \pm 8.1^{*}$ & $<0.05$ & $38.5 \pm 6.8^{*}$ & $<0.05$ \\
\hline 6 months & 102 & $66.5 \pm 9.4^{\#}$ & $<0.05$ & $50.6 \pm 9.2^{\#}$ & $<0.05$ & $35.6 \pm 8.2^{\#}$ & $<0.05$ \\
\hline 9 months & 100 & $58.8 \pm 10.6^{\&}$ & $<0.05$ & $44.3 \pm 7.9^{\&}$ & $<0.05$ & $32.4 \pm 7.9^{\&}$ & $<0.05$ \\
\hline
\end{tabular}

Note: compared with baseline, * $\mathrm{P}<0.05$; compared with 3 months, ${ }^{*}, \mathrm{P}<0.05$; compared with 6 months, ${ }^{\text {, }}, \mathrm{P}<0.05$; compared with 9 months, ${ }^{\circledR}, \mathrm{P}<0.05$. LVEDD, left ventricular end diastolic diameter; LVESD, left ventricular end systolic diameter; LAD, left atrium diameter; $\mathrm{HFrEF}$, heart failure with reduced ejection fraction.

Table 6 Variation of eGFR and serum potassium from baseline to 12 months in HFrEF treatment with Sacubitril/Valsartan

\begin{tabular}{lcccc}
\hline Time & Number of cases $(\mathrm{n})$ & eGFR & $P$ & Serum potassium \\
\hline Baseline & 120 & $58.6 \pm 22.4$ & - & $4.2 \pm 0.5$ \\
3 months & 105 & $59.3 \pm 20.8$ & $>0.05$ & $4.5 \pm 0.8$ \\
6 months & 102 & $58.2 \pm 19.7$ & $>0.05$ & $4.7 \pm 0.6$ \\
9 months & 100 & $58.0 \pm 18.8$ & $>0.05$ & $4.3 \pm 0.4$ \\
12 months & 98 & $58.8 \pm 19.2^{\circledR}$ & $>0.05$ & $4.6 \pm 0.4$ \\
\hline
\end{tabular}

Note: compared with baseline, ${ }^{\circledR}, \mathrm{P}<0.05$. eGFR, estimated glomerular filtration rate; HFrEF, heart failure with reduced ejection fraction.

immediately after admission. The changes in the cardiac remodeling indices, 6-minute walking distance, KCCQ scores and NT-proBNP concentration levels in the same patient before and after treatment were observed, which further revealed that the causes of improvement included quality of life and prognosis in patients treated with Sacubitril/Valsartan.

Our study found that the average NT-proBNP concentration in the same patients before and after treatment with Sacubitril/Valsartan was significantly reduced by $40 \%$ after 1 -month follow-up, and it showed a continuing and stable decrease after 3-, 6-, 9- and 12 -month's follow-up. After 3 months' treatment with Sacubitril/Valsartan, the mean LVEF value increased, and LVEDD, LVESD, and the LAD decreased. Compared with baseline measures prior to treatment, these differences were statistically significant. Moreover, after 6, 9, and 12 months of observation, the LVEF continued to increase, and the left atrial and left ventricular diameters continued to decrease. Compared with baseline measures prior to treatment, these differences were statistically significant. Moreover, with the decrease of NT-proBNP concentrations and the improvement in the cardiac remodeling index, the patients' quality of life improved (demonstrated by increases in the 6-minute walking test distance and KCCQ quality of life scores). These results are consistent with the research results of the PARADIGM-HF study. After treatment with Sacubitril/Valsartan and other active heart failure drugs, the symptoms of heart failure were improved, and the secretion of NT-proBNP in the body was significantly reduced. Over the prolonged course of treatment, the size of the atrium and ventricles retracted noticeably, indicating that cardiac remodeling had also been inhibited. After cardiac remodeling was inhibited, cardiac function improved, the 6-minute walking distance was extended, the KCCQ scores increased (indicating an improvement in the patients' quality of life), and prognosis was improved.

\section{Conclusions}

This study further confirmed the effectiveness and safety of Sacubitril/Valsartan treatment in patients with HFrEF. In this study, all patients with HFrEF, regardless of prior ACEI/ARB use, were treated with Sacubitril/Valsartan in the early stage after admission, and follow-up data from the same patients after 12 months' treatment showed results 
consistent with the PARADIGM-HF study. Moreover, this result is more applicable to a wider population of $\mathrm{HFrEF}$ patients than the PARADIGM-HF study.

\section{Acknowledgments}

Funding: This study was supported by the social development fund of the Science and Technology Department of Shaanxi Province (2019SF-114).

\section{Footnote}

Reporting Checklist: The authors have completed the TREND reporting checklist. Available at http://dx.doi. org/10.21037/apm-21-157

Data Sharing Statement: Available at http://dx.doi. org/10.21037/apm-21-157

Conflicts of Interest: All authors have completed the ICMJE uniform disclosure form (available at http://dx.doi. org/10.21037/apm-21-157). The authors have no conflicts of interest to declare.

Ethical Statement: The authors are accountable for all aspects of the work in ensuring that questions related to the accuracy or integrity of any part of the work are appropriately investigated and resolved. The study was approved by institutional ethics board of First Affiliated Hospital of Medical College, Xi'an Jiaotong University (No.:2019SF-114). All participants gave written informed consent to participate in this study, and the study protocol conformed to the ethical guidelines of the Declaration of Helsinki (as revised in 2013).

Open Access Statement: This is an Open Access article distributed in accordance with the Creative Commons Attribution-NonCommercial-NoDerivs 4.0 International License (CC BY-NC-ND 4.0), which permits the noncommercial replication and distribution of the article with the strict proviso that no changes or edits are made and the original work is properly cited (including links to both the formal publication through the relevant DOI and the license). See: https://creativecommons.org/licenses/by-nc-nd/4.0/.

\section{References}

1. Chen LM, Levine DA, Hayward R, et al. Relationship between Hospital 30-Day Mortality Rates for Heart Failure and Patterns of Early Inpatient Comfort Care. J Hosp Med 2018;13:170-6.

2. Oka T, Akazawa H, Naito AT, et al. Angiogenesis and cardiac hypertrophy: maintenance of cardiac function and causative roles in heart failure. Circ Res 2014;114:565-71.

3. Zheng X, Wang S, Zou X, et al. Ginsenoside Rb1 improves cardiac function and remodeling in heart failure. Exp Anim 2017;66:217-28.

4. Ma T, Zhu D, Chen D, et al. Sulforaphane, a Natural Isothiocyanate Compound, Improves Cardiac Function and Remodeling by Inhibiting Oxidative Stress and Inflammation in a Rabbit Model of Chronic Heart Failure. Med Sci Monit 2018;24:1473-83.

5. Bertero E, Maack C. Metabolic Remodelling in Heart Failure. Nat Rev Cardiol 2018;15:457-70.

6. Lüscher TF. Heart failure and left ventricular remodelling in HFrEF and HFpEF. Eur Heart J 2016;37:423-4.

7. Yasser K, Victor S, John J, et al. Sacubitril/Valsartan (LCZ696) in Heart Failure. Heart Failure 2016;243:135-65.

8. Solomon SD, Rizkala AR, Gong J, et al. Angiotensin Receptor Neprilysin Inhibition in Heart Failure With Preserved Ejection Fraction: Rationale and Design of the PARAGON-HF Trial. JACC Heart Fail 2017;5:471-82.

9. Campbell RT, Petrie MC, Jackson CE, et al. Which patients with heart failure should receive specialist palliative care? Eur J Heart Fail 2018;20:1338-47.

10. Yancy CW, Jessup M, Bozkurt B, et al. 2017 ACC/ AHA/HFSA Focused Update of the 2013 ACCF/AHA Guideline for the Management of Heart Failure: A Report of the American College of Cardiology/American Heart Association Task Force on Clinical Practice Guidelines and the Heart Failure Society of America. J AM Coll Cardiol 2017;70:776-803.

11. Ahmeti A, Henein MY, Ibrahimi P, et al. Quality of life questionnaire predicts poor exercise capacity only in HFpEF and not in HFrEF. BMC Cardiovascular Disorders 2017;17:268-78.

12. Pogge EK, Davis LE, et al. Evaluating the Safety and Tolerability of Sacubitril/Valsartan for HFrEF Managed within a Pharmacist Clinic. Am J Cardiovasc Drugs 2018;18:143-51.

13. Goldsmith SR, Udelson JE, Gheorghiade M. Dual Vasopressin V1a/V2 Antagonism: The Next Step in Neurohormonal Modulation in Patients With Heart Failure? J Card Fail 2018;24:112-4.

14. Khan MS, Siddiqi TJ, Usman MS, et al. Does natriuretic peptide monitoring improve outcomes in heart failure 
patients? A systematic review and meta-analysis. Int J Cardiol 2018;263:80-7.

15. Shah KS, Xu H, Matsouaka RA, et al. Heart Failure With Preserved, Borderline, and Reduced Ejection Fraction: 5-Year Outcomes. J Am Coll Cardiol 2017;70:2476-86.

16. Li Y, Li Z, Zhang C, et al. Cardiac Fibroblast-Specific Activating Transcription Factor 3 Protects Against Heart Failure by Suppressing MAP2K3-p38 Signaling. Circulation 2017;135:2041-57.

17. Gori M, Volterrani M, Piepoli M, et al. Angiotensin receptor-neprilysin inhibitor (ARNi): Clinical studies on a new class of drugs. Int J Cardiol 2017;226:136-40.

18. Lewis GA, Schelbert EB, Williams SG, et al. Biological phenotypes of heart failure with pre-served ejection fraction. J Am Coll Cardiol 2017;70:2186-200.

19. Mogensen UM, Køber L, Kristensen SL, et al. The

Cite this article as: Chen F, Tian G, Bai X, Li J, Yuan Z. The effect of Sacubitril/Valsartan on cardiac function and cardiac remodeling in patients with heart failure with reduced ejection fraction. Ann Palliat Med 2021;10(8):8684-8691. doi: 10.21037/ apm-21-157
Effects of Sacubitril/Valsartan on Coronary Outcomes in PARADIGM-HF. Am Heart J 2017;188:35-41.

20. Martens P, Belien H, Dupont M, et al. Insights into implementation of sacubitril/valsartan into clinical practice. ESC Heart Fail 2018;5:275-83.

21. Luo N, Fonarow GC, Lippmann SJ, et al. Early Adoption of Sacubitril/Valsartan for Patients With Heart Failure With Reduced Ejection Fraction: Insights From Get With the Guidelines-Heart Failure (GWTG-HF). JACC Heart Fail 2017;5:305-9.

22. DeVore AD, Thomas L, Albert NM, et al. Change the management of patients with heart failure: Rationale and design of the CHAMP-HF registry. Am Heart J 2017;189:177-83.

(English Language Editor: D. Fitzgerald) 